\title{
Finite-Level Quantized Feedback Control for Linear Systems
}

\author{
Minyue $\mathrm{Fu}$ and Lihua Xie
}

\begin{abstract}
Recent studies have shown that a logarithmic quantizer can provide the coarsest quantization for quadratic stabilization of linear systems using quantized feedback. However, the coarsest quantizer has an infinite number of quantization levels, which is not implementable in practice. In this paper, we investigate the quantized feedback control problem for discrete-time linear systems using a finite-level logarithmic quantizer. We introduce a dynamic scaling method for the logarithmic quantizer and show that asymptotic stabilization can be achieved with a moderate number of quantization levels. Our approach is easily implementable. We also study the quantized feedback stabilization problem for systems with bounded stochastic noise inputs and show that the system state can converge to a bounded region using a finite-level logarithmic quantizer in conjunction with a proper dynamic scaling scheme.
\end{abstract}

\section{INTRODUCTION}

There has been a lot of resurgent interest in quantized feedback control recently. Unlike previous research where the interest was on the digital implementation of control systems, the recent interest focuses on the use of digital communication channels for feedback control. In this setting, the feedback signal is quantized and then coded for transmission. From the control design point of view, a fundamental problem is how to design a feedback controller and a quantizer jointly in order to achieve a given control objective.

The research on quantized feedback control can be categorized depending on whether the quantizer is static or dynamic. A static quantizer is a memoryless nonlinear function, whereas a dynamic quantizer uses memory and thus can be much more complex and potentially more powerful. Existing work using static quantizers includes, e.g., [1], [2], [3], [14]. For quadratic stabilization of a linear system using state feedback, it is shown in [1] that the optimal static quantizer is a logarithmic quantizer. This result is generalized in [3] to a number of output feedback problems using a sector bound approach, where logarithmic quantizers are also shown to be optimal.

When a dynamic quantizer is allowed, it is shown in [4] that stabilization of a SISO LTI system (in some stochastic sense) can be achieved using only a finite number of quantization levels, and the minimum number of quantization levels (also known as the minimum feedback information rate) is explicitly related to the unstable poles of the system. In this setting, the dynamic quantizer effectively consists of two

Minyue $\mathrm{Fu}$ is with School of EE\&CS, University of Newcastle, Callaghan, N.S.W. 2308 Australia (email: minyue.fu@ newcastle.edu.au).

Lihua Xie is with School of EEE, Nanyang Technological University, Singapore (email: elhxie@ntu.edu.sg). parts: an encoder at the output end and a decoder at the input end.

Another type of dynamic quantizers uses dynamic scaling in conjunction with a static quantizer. That is, the input signal is pre-scaled so that its range is more suitable for quantization. The scaling parameter is dynamically adjusted (i.e., adjusted online). Noticeable work along this line includes [9]-[12]. In [9], it is pointed out that if a system is not excessively unstable, by employing a quantizer with various sensitivity a feedback strategy can be designed to bring the closed-loop state arbitrarily close to zero for an arbitrarily long time. The idea of quantizer with sensitivity is extended in [10] where it is shown that there exists a dynamic adjustment of the quantizer sensitivity and a quantized state feedback that asymptotically stabilizes the system. In the case of output feedback, a local (or semi-global) stabilization result is obtained. We note that dynamic scaling is a very popular idea in signal processing for reducing quantization errors [13] but the key difference here is that we also need to guarantee stability in control.

This paper is primarily inspired by the work of [4] but also motivated by its limitations. Although it is shown in [4] that stabilization of a linear system can be achieved by feeding back only a finite number of bits per sample and this number is typically very small, the encoder-decoder scheme used for proving this result is impractical and non-robust. It is impractical because the encoder-decoder pair is a very nonlinear operator which would typically result in a large overshoot, and it can be non-robust because even a very small amount of noise in the system can drive the closed-loop unstable. These problems are not present in a logarithmic quantizer based feedback controller because the quantization error can be modeled as a sector bounded uncertainty and the closed-loop system is essentially linear. The main drawback of the logarithmic quantizer is that it requires an infinite number of quantization levels.

In this paper, we propose a simple dynamic scaling method for a logarithmic quantizer based feedback controller. A dynamic scaling factor is simply adjusted up or down depending whether the input signal to the quantizer is "too small" or "too large". Using this dynamic scaling method, we show that a linear system can be asymptotically stabilized using a logarithmic quantizer with only a finite number of quantization levels. This number turns out to be very moderate (typically a few bits to a few bytes) and is usually very compatible to the minimum information rate given in [4]. The main advantage of the proposed scheme is that the system behaves as if there were an infinite number of logarithmic 
quantization levels when the initial state is "moderate" in size, i.e., the state would converge exponentially. Only when the initial state is very large, a transient period of overshoot can be present. The region of exponential convergence can be easily increased by using more quantization levels, and the number of feedback information bits grows only at a $\log (\log (\cdot))$ rate when the size of this region increases. Since most digital communication channels can easily handle a few tens of bytes per sample, the proposed scheme should be very practical. We also show that the proposed scheme is robust in the sense it can tolerate additive noises in the system effectively.

\section{INFINITE-LEVEL LOGARITHMIC QUANTIZATION}

Consider the following system:

$$
\begin{aligned}
x_{k+1} & =A x_{k}+B u_{k}, \\
y_{k} & =C x_{k},
\end{aligned}
$$

where $x_{k} \in \mathbb{R}^{n}$ is the state, $u_{k} \in \mathbb{R}$ is the control input, $y_{k} \in \mathbb{R}$ is the measured output, $A \in \mathbb{R}^{n \times n}, B \in \mathbb{R}^{n \times 1}$ and $C \in \mathbb{R}^{1 \times n}$ are given. We will denote the transfer function from $u_{k}$ to $y_{k}$ by $G(z)$. Without loss of generality, we assume that $A$ is unstable and $(A, B, C)$ is a minimal realization.

The quantized feedback control problem is to design a feedback quantizer

$$
v_{k}=Q\left(y_{k}\right)
$$

which takes values in

$$
\mathcal{V}=\left\{ \pm \mu_{i}: i=0, \pm 1, \pm 2, \cdots\right\} \cup\{0\},
$$

and a feedback controller of the form

$$
\begin{aligned}
\hat{x}_{k+1} & =A_{c} \hat{x}_{k}+B_{c} v_{k}, \quad \hat{x}_{0}=0, \\
u_{k} & =C_{c} \hat{x}_{k}+D_{c} v_{k},
\end{aligned}
$$

with $\hat{x}_{k} \in \mathbb{R}^{n}$, such that the closed-loop system is stable and that the so-called quantization density [1] is coarsest. The quantization density of $Q(\cdot)$ is defined as follows:

$$
\eta_{Q}=\lim \sup _{\epsilon \rightarrow 0} \frac{\# g[\epsilon]}{-\ln \epsilon}
$$

where $\# g[\epsilon]$ denotes the number of quantization levels in the interval $[\epsilon, 1 / \epsilon]$.

The quantized feedback control problem for the system (1)-(2) is generalized from a quantized state feedback control problem in [1] and has been studied in details in [3]. In particular, it is known [3] that the coarsest quantization density for quadratic stabilization of the system above is achieved by a logarithmic quantizer. Such a quantizer is described by

$$
\mathcal{V}=\left\{\mu_{i}=\rho^{i} \mu_{0}: i=0, \pm 1, \pm 2, \cdots\right\} \cup\{0\}, \mu_{0}>0,
$$

where $\rho \in(0,1)$. Since a smaller $\rho$ corresponds to a smaller $\eta_{Q}$, we can regard $\rho$ as the quantization density instead. The associated quantizer $Q(\cdot)$ is defined as follows:

$$
Q(y)= \begin{cases}\rho^{i} \mu_{0}, & \text { if } \frac{1}{1+\delta} \rho^{i} \mu_{0}<y \leq \frac{1}{1-\delta} \rho^{i} \mu_{0} \\ 0, & \text { if } y=0 \\ -Q(-y), & \text { if } y<0,\end{cases}
$$

where

$$
\delta=(1-\rho)(1+\rho)^{-1} .
$$

It is further shown in [3] that the smallest $\rho$ for which the system (1)-(2) can be quadratically stabilized via a quantized feedback controller (3), (5)-(6) is given by

$$
\begin{aligned}
\rho_{\text {inf }} & =\frac{1-\delta_{\text {sup }}}{1+\delta_{\text {sup }}}, \\
\delta_{\text {sup }}^{-1} & =\inf _{H(z)}\left\|(1-H(z) G(z))^{-1} H(z) G(z)\right\|_{\infty},
\end{aligned}
$$

where $H(z)$ is the transfer function of the controller.

\section{Finite-Level Quantized Feedback STABILIZATION}

It is obvious that a logarithmic quantizer (9) has an infinite number of quantization levels. This is certainly not implementable practically. One simple approach is to truncate the quantizer using a large saturator and a small dead zone. This will allow the state of the system to converge to a small neighborhood, provided that the initial state is within a known bound. Due to the use of logarithmic quantization, the number of quantization levels required is far less than required by using linear quantization.

In this section, we show that it is possible to dynamically scale the input-output signals of the quantizer so that asymptotic stabilization can be achieved using a finite-level logarithmic quantizer, even without knowing the bound for the initial state. We define an $N$-level logarithmic quantization with quantization density $\rho>\rho_{\text {inf }}$ as

$$
\mathcal{V}=\left\{ \pm \rho^{i} \mu_{0}, i=0,1,2, \cdots, N-1\right\}, \quad \mu_{0}>0 .
$$

The associated quantizer $Q(\cdot)$ becomes:

$$
Q(y)= \begin{cases}\rho^{i} \mu_{0}, & \text { if } \frac{1}{1+\delta} \rho^{i} \mu_{0}<y \leq \frac{1}{1-\delta} \rho^{i} \mu_{0}, \\ \rho^{N-1} \mu_{0}, & \text { if } 0 \leq y \leq \frac{1}{1+\delta} \rho^{N-1} \mu_{0}, \\ \mu_{0}, & \text { if } y>\frac{1}{1-\delta} \rho^{i} \mu_{0}, \\ -Q(-y), & \text { if } y<0 .\end{cases}
$$

The basic idea of dynamic scaling is very simple: When the signal $y_{k}$ is outside of the quantization range, we scale it back by a scaling factor (or gain) $g_{k}>0$ before quantization. The quantized signal is then scaled back by $g_{k}^{-1}$. That is, we use

$$
v_{k}=g_{k}^{-1} Q\left(g_{k} y_{k}\right) .
$$

Suppose an infinite-level logarithmic quantizer with density $\rho>\rho_{\text {inf }}$ is adopted. Following the sector bound approach [3], we can write the closed-loop system of (1)(2) and (5)-(6) as

$$
\begin{aligned}
\xi_{k+1} & =\bar{A}\left(\Delta_{k}\right) \xi_{k} \\
y_{k} & =\bar{C} \xi_{k}
\end{aligned}
$$


where $\xi=\left[\begin{array}{ll}x^{T} & \hat{x}^{T}\end{array}\right]^{T}$,

$$
\begin{gathered}
\bar{A}\left(\Delta_{k}\right)=\bar{A}+\bar{B}\left(1+\Delta_{k}\right) \bar{C} \\
\bar{A}=\left[\begin{array}{cc}
A & B C_{c} \\
0 & A_{c}
\end{array}\right], \quad \bar{B}=\left[\begin{array}{c}
B D_{c} \\
B_{c}
\end{array}\right], \quad \bar{C}=\left[\begin{array}{ll}
C & 0
\end{array}\right] .
\end{gathered}
$$

Also,

$$
\Delta_{k} y_{k}=Q\left(y_{k}\right)-y_{k}, \quad\left|\Delta_{k}\right| \leq \delta,
$$

represents the quantization error. Because (16) is quadratically stable, we have a quadratic Lyapunov function $V(\xi)=$ $\xi^{T} P \xi$ with $P=P^{T}>0$ such that

$$
V\left(\xi_{k+1}\right)-V\left(\xi_{k}\right)=\xi_{k}^{T}\left(\bar{A}\left(\Delta_{k}\right)^{T} P \bar{A}\left(\Delta_{k}\right)-P\right) \xi_{k}<0
$$

for all nonzero $\xi_{k} \in \mathbb{R}^{2 n}$ and admissible $\Delta_{k}$. It is shown in [3] that the above is equivalent to

$$
\bar{A}(\Delta)^{T} P \bar{A}(\Delta)-P<0, \quad \forall|\Delta| \leq \delta .
$$

Using the continuity argument, the above is equivalent to

$$
\bar{A}(\Delta)^{T} P \bar{A}(\Delta)-P \leq-\eta P, \quad \forall|\Delta| \leq \delta,
$$

for some $0<\eta<1$.

We now assume that an $N$-level logarithmic quantizer with the same density $\rho$ and dynamic scaling (15) is applied instead. We choose two positive scaling factors $0<\gamma_{1}, \gamma_{2}<$ 1 such that

$$
\begin{gathered}
\gamma_{1}^{2} \bar{A}^{T} P \bar{A}-P<-\eta_{1} P, \\
\gamma_{2}^{-2}(1+\tau) \bar{A}(\Delta)^{T} P \bar{A}(\Delta)-P<-\eta_{2} P, \quad \forall\|\Delta\| \leq \delta,
\end{gathered}
$$

for some $0<\eta_{1}, \eta_{2}, \tau<1$. The latter is done by choosing $\gamma_{2}$ close to 1 and $\tau$ close to 0 so that $\gamma_{2}^{-2}(1+\tau)(1-\eta)<1$ and taking

$$
\eta_{2}=1-\gamma_{2}^{-2}(1+\tau)(1-\eta) .
$$

This ensures $\eta_{2}>0$ and makes (24) equivalent to (22).

We initialize $g_{0}$ to be any positive value and define $g_{k+1}$ for any $k \geq 0$ as follows:

$$
g_{k+1}= \begin{cases}g_{k} \gamma_{1}, & \text { if }\left|Q\left(g_{k} y_{k}\right)\right|=\mu_{0}, \\ g_{k} / \gamma_{2}, & \text { if }\left|Q\left(g_{k} y_{k}\right)\right|=\rho^{N-1} \mu_{0} \\ g_{k}, & \text { otherwise. }\end{cases}
$$

Because of the flexibility in $g_{0}$, we can normalize $\mu_{0}=1$ without loss of generality. We will also denote $\bar{\varepsilon}=\rho^{N-1}$. The choice of $g_{0}$ does not affect stabilizability, but choosing it according to an estimate of $\left\|x_{0}\right\|$ helps improve the transient performance.

To help analyze the quantized feedback system, we consider the scaled state defined by

$$
z_{k}=g_{k} \xi_{k}
$$

and the associated Lyapunov function $V(z)=z^{T} P z$. We have the following result:
Lemma 3.1: Suppose the scaled N-level logarithmic quantizer (14), (15) and (26) is applied. Then, for any initial state $x_{0}$ and any $k \geq 0$,

$$
\begin{aligned}
& V\left(z_{k+1}\right)-V\left(z_{k}\right) \\
& \leq \begin{cases}-\eta_{3} V\left(z_{k}\right), & \text { if }\left|Q\left(\bar{C} z_{k}\right)\right|=1, \\
-\eta V\left(z_{k}\right), & \text { if } \bar{\varepsilon}<\left|Q\left(\bar{C} z_{k}\right)\right|<1, \\
-\eta_{2} V\left(z_{k}\right)+\eta_{4} \bar{\varepsilon}^{2}, & \text { if }\left|Q\left(\bar{C} z_{k}\right)\right|=\bar{\varepsilon},\end{cases}
\end{aligned}
$$

where

$$
\begin{aligned}
\eta_{3} & =\max \left\{\eta_{1}, 1-\gamma_{1}^{2}(1-\eta)\right\}>0 \\
\eta_{4} & =\gamma_{2}^{-2}\left(1+\tau^{-1}\right) \bar{B}^{T} P \bar{B}
\end{aligned}
$$

Proof: The result for the case of $\rho^{N-1}<\left|Q\left(\bar{C} z_{k}\right)\right|<1$ follows directly from (16), (22) and $g_{k+1}=g_{k}$. For the case of $\left|Q\left(\bar{C} z_{k}\right)\right|=1, g_{k+1}=g_{k} \gamma_{1}$. It follows that

$V\left(z_{k+1}\right)-V\left(z_{k}\right)=\gamma_{1}^{2}\left(\bar{A} z_{k}+\bar{B} \sigma_{k}\right)^{T} P\left(\bar{A} z_{k}+\bar{B} \sigma_{k}\right)-z_{k}^{T} P z_{k}$, where $\sigma_{k}=\operatorname{sign}\left(\bar{C} z_{k}\right)$. Denote

$$
f(u)=\gamma_{1}^{2}\left(\bar{A} z_{k}+\bar{B} u\right)^{T} P\left(\bar{A} z_{k}+\bar{B} u\right)-z_{k}^{T} P z_{k} .
$$

From (23),

$$
f(0) \leq-\eta_{1} z_{k}^{T} P z_{k} .
$$

Since $\sigma_{k}=Q\left(\bar{C} z_{k}\right)$, we have $\sigma_{k}=\theta u_{1}$ for some $0<\theta \leq 1$, where $u_{1}=\left(1+\Delta_{k}\right) \bar{C} z_{k}$ is the unsaturated output of the quantizer. Also from (22), we get

$$
\begin{aligned}
f\left(u_{1}\right) & =\gamma_{1}^{2} z_{k}^{T} \bar{A}\left(\Delta_{k}\right)^{T} P \bar{A}\left(\Delta_{k}\right) z_{k}-z_{k}^{T} P z_{k} \\
& \leq-\left(1-\gamma_{1}^{2}(1-\eta)\right) V\left(z_{k}\right) .
\end{aligned}
$$

Since $f(u)$ is quadratic and convex (because $f(u) \rightarrow+\infty$ when $|u| \rightarrow \infty)$, it is clear that

$$
\begin{aligned}
V\left(z_{k+1}\right)-V\left(z_{k}\right) & =f\left(\sigma_{k}\right) \leq \max \left\{f(0), \quad f\left(u_{1}\right)\right\} \\
& =-\eta_{3} V\left(z_{k}\right) .
\end{aligned}
$$

For the case of $\left|Q\left(\bar{C} z_{k}\right)\right|=\bar{\varepsilon}, g_{k+1}=g_{k} / \gamma_{2}$. From (13) and (16), we can write

$$
x_{k+1}=\bar{A}\left(\Delta_{k}\right) x_{k}+\bar{B} g_{k}^{-1} \varepsilon_{k},
$$

where $\left|\varepsilon_{k}\right| \leq \bar{\varepsilon}$. It follows that

$$
\begin{aligned}
& V\left(z_{k+1}\right)-V\left(z_{k}\right) \\
= & \gamma_{2}^{-2}\left(\bar{A}\left(\Delta_{k}\right) z_{k}+\bar{B} \varepsilon_{k}\right)^{T} P\left(\bar{A}\left(\Delta_{k}\right) z_{k}+\bar{B} \varepsilon_{k}\right) \\
& -z_{k}^{T} P z_{k} \\
= & \gamma_{2}^{-2} z_{k}^{T} \bar{A}\left(\Delta_{k}\right) P \bar{A}\left(\Delta_{k}\right) z_{k}-z_{k}^{T} P z_{k} \\
& +\gamma_{2}^{-2}\left(2 \varepsilon_{k} \bar{B}^{T} P \bar{A}\left(\Delta_{k}\right) z_{k}+\varepsilon_{k}^{2} \bar{B}^{T} P \bar{B}\right) \\
\leq & \gamma_{2}^{-2}(1+\tau) z_{k}^{T} \bar{A}\left(\Delta_{k}\right) P \bar{A}\left(\Delta_{k}\right) z_{k}-z_{k}^{T} P z_{k} \\
& +\gamma_{2}^{-2}\left(1+\tau^{-1}\right) \bar{\varepsilon}^{2} \bar{B}^{T} P \bar{B} \\
= & -\eta_{2} z_{k}^{T} P z_{k}+\eta_{4} \bar{\varepsilon}^{2} .
\end{aligned}
$$

This completes the proof.

From Lemma 3.1, it is clear that $V\left(z_{k}\right)$ converges to a bounded region. This bound can be computed by solving

$$
0=-\eta_{2} V_{\infty}+\eta_{4} \bar{\varepsilon}^{2},
$$


which gives

$$
V_{\infty}=\eta_{2}^{-1} \eta_{4} \bar{\varepsilon}^{2}
$$

Lemma 3.1 leads to the following result:

Corollary 3.1: Suppose the scaled N-level logarithmic quantizer (14), (15) and (26) is applied. Then, for any initial state $x_{0}, z_{k}=g_{k} \xi_{k}$ converges exponentially to the ellipsoid

$$
Z_{\infty}=\left\{z: z \in \mathbb{R}^{2 n}, V(z) \leq V_{\infty}\right\}
$$

From (30) and the corollary above, it is clear that we can choose $N$ to be sufficiently large so that, when $k$ is sufficiently large, $Q\left(\bar{C} z_{k}\right)$ will no longer be saturated. This is achieved by choosing $N$ such that

$$
|\bar{C} z|<1 \quad \forall z^{T} P z \leq \eta_{2}^{-1} \eta_{4} \rho^{2(N-1)} .
$$

Solving this gives $N \geq N_{0}$, where

$$
N_{0}=1+\frac{\log \left(\eta_{2}^{-1} \gamma_{2}^{-2}\left(1+\tau^{-1}\right) \bar{B}^{T} P \bar{B} \bar{C} P^{-1} \bar{C}^{T}\right)}{2 \log \left(\rho^{-1}\right)} .
$$

The analysis above yields the following main result:

Theorem 3.1: Suppose the scaled N-level logarithmic quantizer (14), (15) and (26) is applied with $N \geq N_{0}$ in (32). Then, the state $\xi_{k}$ converges to zero asymptotically.

Proof: From Corollary 3.1, $z_{k}$ converges to $Z_{\infty}$ exponentially. This property and the choice of $N_{0}$ imply that $Q\left(\bar{C} z_{k}\right)$ will no longer be saturated after a finite number of steps, say $k_{0}$ steps. This means that $g_{k}$ will be non-decreasing for $k \geq k_{0}$. Note that whenever $g_{k+1}=g_{k}, V\left(z_{k}\right)$ decreases exponentially. If this continues for enough number of steps, $\left|\bar{C} z_{k}\right|$ be less than $\bar{\epsilon}$, forcing $g_{k+1}$ to increase by factor of $1 / \gamma_{2}$. This means that $g_{k}$ cannot converge to a constant. Hence, $g_{k} \rightarrow \infty$ as $k \rightarrow \infty$. Since $z_{k}$ is bounded for $k>k_{0}$, we conclude that $\xi_{k} \rightarrow 0$ as $k \rightarrow \infty$.

Remark 3.1: A typical behavior of the system is as follows: If the initial state is very large, the feedback signal tends to be saturated, forcing $g_{k}$ to decrease fast. This would result in a period of overshoot. Once $g_{k}$ is sufficiently small, saturation will stop and the state decays exponentially. When the state is sufficiently small, $g_{k}$ will increase gradually, causing the quantizer to bounce back and forth between the dead zone and logarithmic region. During this phase, the state also decays exponentially, but at a lower rate.

Remark 3.2: We note that the number of bits required to code an $N$-level logarithmic quantizer is

$$
N_{b}=\left\lceil\log _{2}(2 N)\right\rceil, \quad N \geq\left\lceil N_{0}\right\rceil,
$$

where $\lceil\cdot\rceil$ is the integer round-up function. This is because there are $N$ levels for both positive and negative inputs.

We can also relate $N_{b}$ to the region of exponential convergence. Since the state $x_{k}$ converges exponentially inside $V_{\infty}$ and $N$ is a logarithmic function of $V_{\infty}$, we know that the number of required information bits grows at a $\log (\log (\cdot))$ rate as the region of exponential convergence for the state increases.

\section{NUMBER OF QUANTIZATION LEVELS}

In this section, we try to analyze the number of quantization levels needed for stabilization. Recall that for a given controller (5)-(6) and an infinite-level logarithmic quantizer with density $\rho>\rho_{\text {inf }}$ that quadratically stabilizes the system (1)-(2), this number is bounded by $N_{0}$ in (32). This formula is complicated because $N_{0}$ depends on a number of design parameters $\left(\eta_{2}, \gamma_{2}, \tau, \rho\right.$ and $\left.P\right)$ which are interconnected.

By minimizing $N_{0}$ with respect to $\tau$ and $\eta_{2}$, we obtain (without showing the details)

$$
\eta_{2}=1-\gamma_{2}^{-1} \sqrt{1-\eta}, \quad \tau=\frac{\gamma_{2}}{\sqrt{1-\eta}}-1
$$

which yields

$$
N_{0}=1+\frac{2 \log \left(\gamma_{2}-\sqrt{1-\eta}\right)-\log \left(\bar{B}^{T} P \bar{B} \bar{C} P^{-1} \bar{C}^{T}\right)}{2 \log (\rho)} .
$$

Since $\gamma_{2}<1$ is required, $N_{0}$ is minimized by taking $\gamma_{2}$ very close to 1 . Although this minimizes $N_{0}$, it will make $g_{k}$ increase very slowly which in turn make $\xi_{k}$ converge to 0 very slowly. A good choice for $\gamma_{2}$ should balance the convergence rate of $\xi_{k}$ and the number of quantization levels; see Example 1 in Section VI. Once $\gamma_{2}$ is chosen, the parameters $\eta$ and $\delta$ need to be optimized jointly. We propose the following simple numerical search method for optimizing $N_{0}$ : Start with any $0<\delta<\delta_{\text {sup }}$ and $0<\eta<1$. Find a controller to satisfy the control objectives. If a satisfactory controller is found, compute the corresponding matrix $P$ and then $N_{0}$. Repeat the above for different values of $\delta$ and $\eta$ and retain the smallest value of $N_{0}$.

\section{Robustness Against Additive Noises}

Next, we consider the scenario where the system (1)-(2) is subject to some bounded additive noise, i.e., we consider the following system instead:

$$
\begin{aligned}
x_{k+1} & =A x_{k}+B u_{k}+w_{k}, \\
y_{k} & =C x_{k}
\end{aligned}
$$

where $\left\|w_{k}\right\| \leq \bar{w}$ for some constant $\bar{w}>0$.

In this case, it is obviously not feasible to drive the state $x_{k}$ to zero. Instead, we want to drive the state to a bounded region. Because of this, there is no need to drive $g_{k}$ to infinity. It turns out that by saturating $g_{k}$ at some upper bound, we can guarantee that the state is driven to a bounded region. This is detailed in the next result (without proof).

Theorem 5.1: Consider the system (36)-(37) and the dynamically scaled logarithmic controller as given before. We modify the scaling factor $g_{k}$ by saturating it at some $\bar{g}>1$. Then, both the closed-loop system state $\xi_{k}$ and the scaled state $z_{k}=g_{k} \xi_{k}$ are bounded when $k \rightarrow \infty$. 


\section{ILLUSTRATIVE EXAMPLES}

In this section, we use several examples to illustrate the proposed dynamic scaling method.

Example 1: We consider a first order system:

$$
\begin{aligned}
x_{k+1} & =a x_{k}+u_{k}, \\
y_{k} & =x_{k},
\end{aligned}
$$

where $a>1$. It turns out that we can have a relatively simple expression for $N_{0}$. Indeed, to stabilize the system using a logarithmic quantizer (9) with density $\rho$, the controller $H(z)=h$ a constant because of full state feedback. The closed-loop system is given by

$$
x_{k+1}=\left(a+h\left(1+\Delta_{k}\right)\right) x_{k}, \quad\left|\Delta_{k}\right| \leq \delta,
$$

where $\delta$ relates to $\rho$ as in (10). Since it is a first order system, we take $V\left(x_{k}\right)=x_{k}^{2}$, which gives

$$
V\left(x_{k+1}\right)=\left(a+h\left(1+\Delta_{k}\right)\right)^{2} x_{k}^{2} \leq(|a+h|+\delta|h|)^{2} x_{k}^{2}
$$

with the right-hand side being the worst-case value. Minimizing it gives $h=-a$ and

$$
V\left(x_{k+1}\right) \leq \delta^{2} a^{2} V\left(x_{k}\right) .
$$

This gives the upper bound for $\delta$ to be $a^{-1}$.

Now, for any $\delta<a^{-1}, \eta$ in (22) is given by $\eta=1-\delta^{2} a^{2}$. Applying it to (35), we obtain

$$
N_{0}=1+\frac{\log \left(\gamma_{2} a^{-1}-\delta\right)}{\log (1-\delta)-\log (1+\delta)}, \delta<a^{-1},
$$

which can be minimized numerically. The result is shown in Figure 1, where two curves for the required bit rate (33), one for $\gamma_{2}=1$ and another for $\gamma_{2}=0.9$, are compared with the minimum bit rate $\left\lceil\log _{2}(a)\right\rceil$ given in [4]. We see that the difference is only a few bits even when $a$ is taken up to 100 .

Example 2: The second example we consider aims at demonstrating the convergence rate and robustness of the dynamic scaling method. Consider the system (1)-(2) with

$$
\begin{aligned}
& A=\left[\begin{array}{ccc}
2.7 & -2.41 & 0.507 \\
1 & 0 & 0 \\
0 & 1 & 0
\end{array}\right], B=\left[\begin{array}{l}
1 \\
0 \\
0
\end{array}\right], \\
& C=\left[\begin{array}{lll}
1 & -0.5 & 0.04
\end{array}\right] .
\end{aligned}
$$

The system is unstable with two unstable open-loop poles at $1.2 \pm i 0.5$ but without unstable zero and the relative degree is 1. It follows from [3] that

$$
\delta_{\text {sup }}=|1.2 \pm i 0.5|^{-2}=0.5917, \quad \rho_{\text {inf }}=0.2565 \text {. }
$$

Choosing $\delta=0.2$ and optimizing $N_{0}$ using a search on $\eta$, we obtain $\eta=0.41$ and the corresponding optimal controller

$$
\begin{aligned}
& A_{c}=\left[\begin{array}{rrr}
0 & 0.5367 & 1.2080 \\
0.1342 & -0.2684 & -0.7382 \\
0.1929 & 0.4194 & 0.1678
\end{array}\right] \text {, } \\
& B_{c}=[1.0000 ; 1.0000 ; 0.3750] \text {, } \\
& C_{c}=\left[\begin{array}{lll}
0.12049 & 0.2293 & -0.1431
\end{array}\right], \\
& D_{c}=-1.4955 \text {. }
\end{aligned}
$$

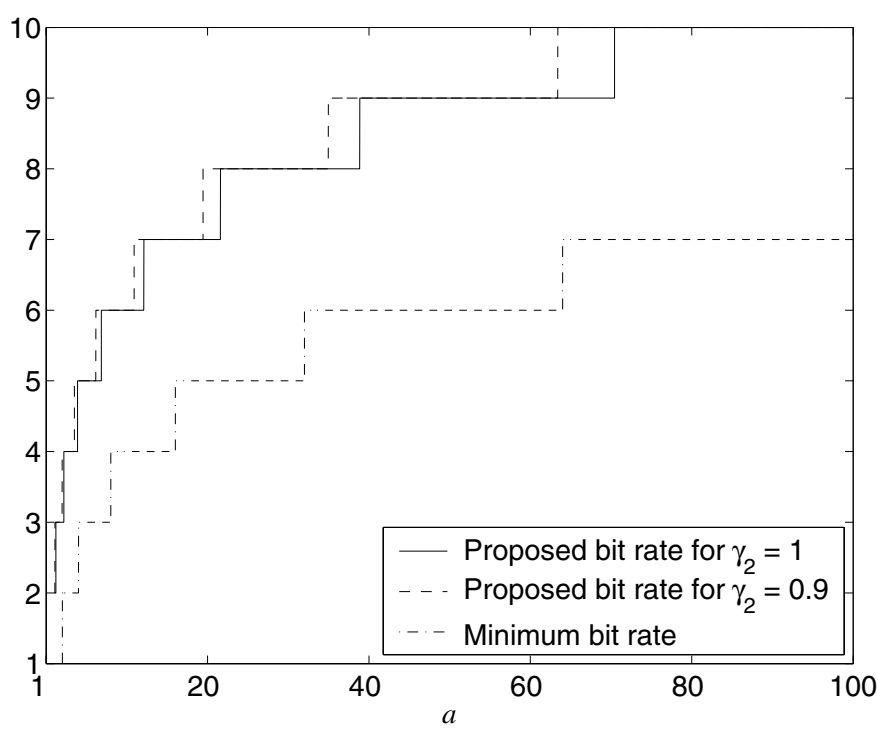

Fig. 1. Bit Rate Comparison for a First Order System

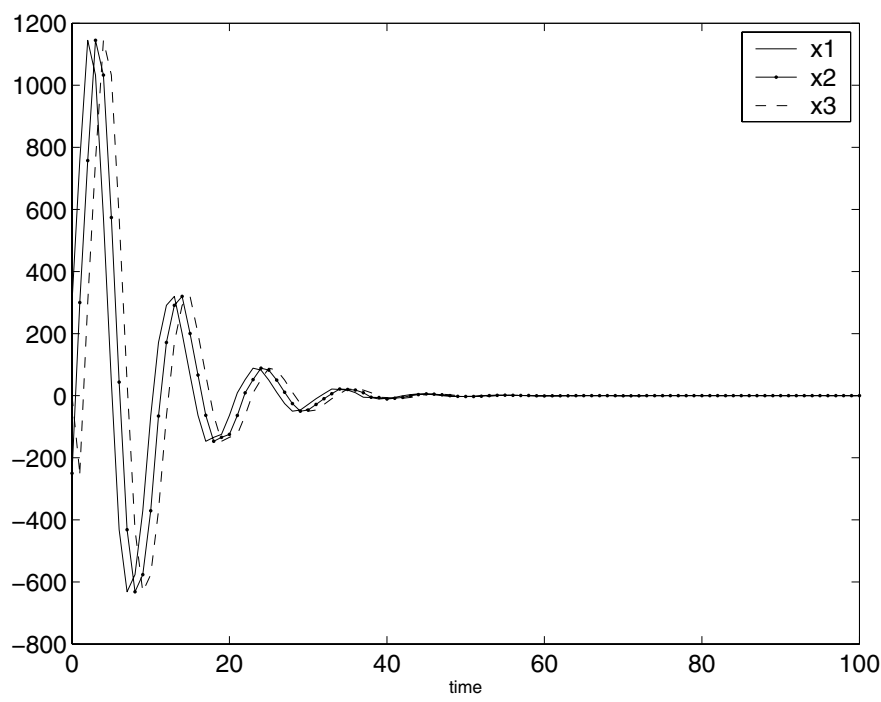

Fig. 2. State response of the closed-loop system

Since $\gamma_{2}$ is lower bounded by $\sqrt{1-\eta}=0.7681$, we choose $\gamma_{2}=0.8$. This gives $N_{0}=11.015$. Since $N=12$ and $N=16$ give the same bit rate ( 5 bits), we set $N=16$. Note that the minimal bit rate required for stabilizing this system is 1 bit [4].

It can be easily verified that (23) is satisfied if we choose $\gamma_{1}=0.2$. Let the initial state of the controller be $\hat{x}_{0}=\left[\begin{array}{lll}0 & 0 & 0\end{array}\right]^{T}$ and the minimal level of the quantizer be 1 (correspondingly, $\mu_{0}=1 / \rho^{N-1}$ ). For the initial state $x_{0}=\left[\begin{array}{lll}250 & -200 & 0\end{array}\right]^{T}$ and $g_{0}=1$, the state response of the closed-loop system is shown in Figure 2. The scaling gain $g_{k}$ is shown in Figure 3.

If we have a good estimate $\tilde{x}_{0}$ of the initial state $x_{0}$, we 


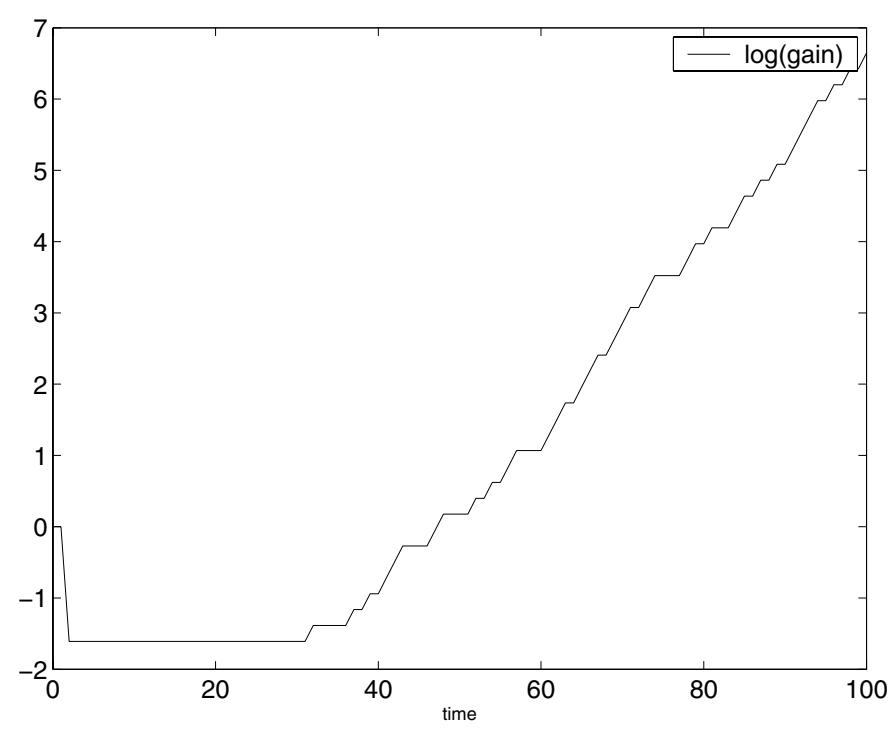

Fig. 3. The scaling factor $g_{k}$ for the noise-free case

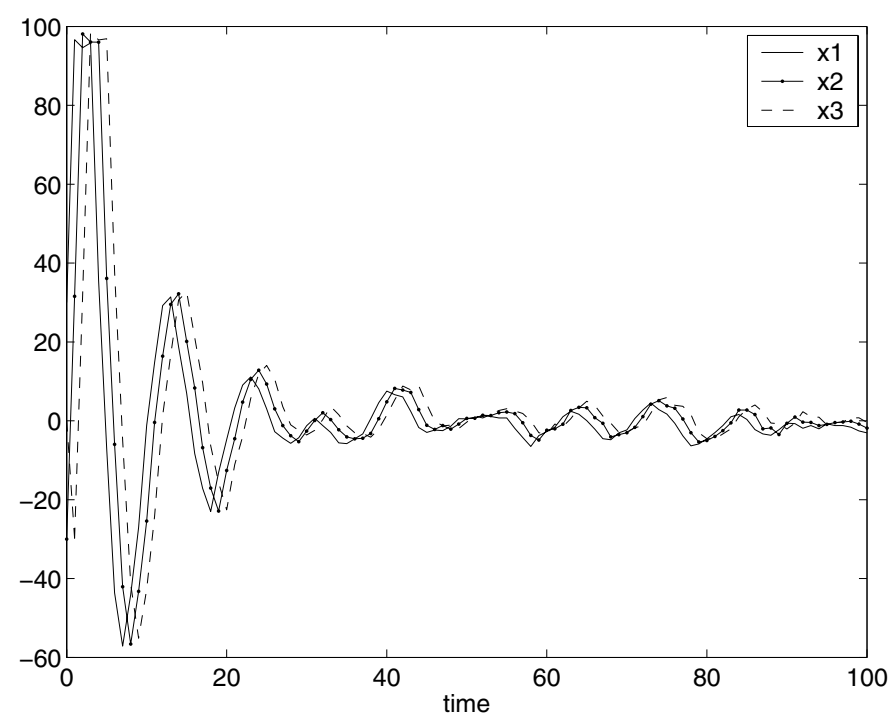

Fig. 4. State response of the closed-loop system under input noise

may set the initial scaling gain $g_{0}$ to improve the transient performance. For example, if we set $g_{0}=1 /\left|C x_{0}\right|$ for the given initial condition, the transient overshoot is reduced by approximately $35 \%$.

Next, we study the robustness of the closed-loop system. Let $w_{k}$ in (36) be a saturated Gaussian white noise with zero mean and $\left|w_{k}\right| \leq 100$ and covariance matrix of $3 I$. We set $\bar{g}=40$. The state response of the closed-loop system with $x_{0}=\left[\begin{array}{lll}30 & -30 & 0\end{array}\right]^{T}$ is shown in Figure 4. It can be observed that the the final state converges to a bounded region. The scaling factor $g_{k}$ has a similar initial response as in the noise free case but gets bounded at the steady state.

\section{CONCLUSION}

We have proposed a simple dynamic scaling method for quantized feedback control. Using this, we show that asymptotic stabilization for a linear system can be achieved using a logarithmic quantizer with a very moderate number of quantization levels. The proposed control scheme is easily implementable and has nice convergence and robust properties. We emphasize that the proposed dynamic scaling method can be applied to a much wider range of quantized feedback control problems.

\section{REFERENCES}

[1] N. Elia and K. Mitter, "Stabilization of Linear Systems with Limited Information," IEEE Transactions on Automatic Control, vol. 46, no. 9, pp. 1384-1400, September, 2001.

[2] N. Elia, "Design of hybrid systems with guaranteed performance," Proc. 39th IEEE Conf. Decision and Control, pp. 993-998, 2000.

[3] M. Fu and L. Xie, "The sector bound approach to quantized feedback control," IEEE Transactions on Automatic Control, vol. 50, no. 11, pp. 1698-1711, Nov. 2005.

[4] G. N. Nair and R. J. Evans, "Exponential stabilization of multidimensional linear systems," Automatica, vol. 39, pp. 585-593, 2003.

[5] G. N. Nair and R. J. Evans, "Stabilization with data-rate-limited feedback: tightest attainable bounds," Systems and Control Letters, vol. 41, pp. 49-56, 2000.

[6] W. S. Wong and R. W. Brockett, "Systems with finite communication bandwidth constraints I: state estimation problems," Transactions on Automatic Control, vol. 42, no. 9, pp. 1294-1299, September, 1997.

[7] W. S. Wong and R. W. Brockett, "Systems with finite communication bandwidth constraints II: stabilization with limited information feedback," Transactions on Automatic Control, vol. 44, no. 5, pp. 10491053 , May, 1999.

[8] J. Baillieul, "Feedback designs in information-based control," Stochastic Theory and Control Workshop, Kansis, pp. 35-57, Springer, 2001.

[9] D. F. Delchamps, "Stabilizing a linear system with qunatized state feedback," IEEE Transactions on Automatic Control, vol. 35, no. 8, pp. 916-924, 1990.

[10] R. W. Brockett and D. Liberzon, "Quantized feedback stabilization of linear systems," IEEE Transactions on Automatic Control, vol. 45, no. 7, pp. 1279-1289, July, 2000.

[11] S. Tatikonda and S. Mitter, "Control under communication constraints," IEEE Transactions on Automatic Control, vol. 49, no. 7, pp. 1056-1068, July, 2004.

[12] S. Tatikonda and S. Mitter, "Control over noisy channels," IEEE Transactions on Automatic Control, vol. 49, no. 7, pp. 1196-1201, July, 2004.

[13] A Mitra, M. Chakraborty and H. Sakai, "A block floating-point treatment to the LMS algorithm: efficient realization and a roundoff eror analysis," IEEE Trans. on Signal Processing, vol. 53, no. 12, 2005.

[14] M. Fu and S. Hara, "Quantized feedback control for sampled-data systems,", IFAC World Congress, Prague, July 2005.

[15] M. C. de Oliveira, J. C. Geromel and J. Bernussou, "An LMI optimization approach to multiobjective controller design for discretetime systems," Proc. the 38th CDC, Phoenix, Dec. 1999.

[16] C. Scherer, P. Gahinet and M. Chilali, "Multiobjective output-feedback control via LMI optimization," IEEE Trans. Automat. Contr., vol. 42, no. 7, pp. 896-911, 1997.

[17] L. Xie and Y. C. Soh, "Guaranteed cost control of uncertain discretetime systems," Control Theory and Advanced Technology, Japan, vol. 10, no. 4, Part 2, pp. 1235-1251, 1995. 